\title{
Twelve Years of Access to Electronic Serials in Russia: Results and Perspectives
}

O. V. Moskaleva ${ }^{l}$, I. K. Razumova ${ }^{2}$

${ }^{1}$ Saint-Petersburg State University. 7-9, Universitetskaya nab., Saint-Petersburg, 199034 Russia

${ }^{2}$ National Electronic Information Consortium. 4(5), Letnikovskaya st. Moscow, 115114 Russia

\begin{abstract}
We investigated collection development within the system of information support of research in Russia on the state-funded level. The analysis has been performed from the point of view of structure and research output of Russian science. We employed the method of bibliometric analysis using InCites by Clarivate Analytics. The results demonstrate that the state funded subscription provides more than $55 \%$ of total world output and meets about $70 \%$ of communication needs of Russian researchers in all ESI research areas. A strong correlation of informational support and research output is demonstrated.
\end{abstract}

Keywords: bibliometric analysis, electronic serials, collection development, library consortia, research output, readership.

\section{Introduction}

In this article we report analysis of the system of information support in Russia.

Indicators of research output such as number of publications and citations are included in national research assessment systems for instance in Austarlia [1], the UK [2], France [3], Italy [4] or Russia [5], and most influential world university rankings. Correlation between the indicators of research output, namely, the number of publications, times cited, share in the world 
output and R\&D funding was studied at the level of individual researchers [6], grant issuing organizations [7] or research-leading countries [8,9], correlation of research output and readership was reported at the level of individual documents within a single journal [10[, institutional [11,12] and country [9] levels. Therefore, one can conclude that problem of information support is one of the key problems in modern research surroundings.

Libraries in academic and research institutions together with departments of research management take active part in handling and analyzing the info on research output, usage statistics, return of investments and impact of scholarly resources on the level of research and teaching. Russia is not an exception and fits world trends of development of information support [13-14]. However, being a country with emerging economy and a member of a group of BRIC countries Russia demonstrates its specific features.

After disintegration of Soviet system in the last decade of XX century, Russia met enormous problems with budget cuttings and brain drain. Finally, Russia has lost its former position among leading countries conducting research in science. In 2006, predictions for the future of Russian research output were grave [15]. In 2005, facing the problem, Russian government launched Federal Target programs aimed at improvement of level of research and education in prioritized research areas of science and technology: Life Sciences; Industry of Nanosystems; ITC Systems; Environmental Management; Energy Efficiency, Energy Saving and Nuclear Energy. From 2006 to 2014 the R\&D budget in Russia was growing and in 2012 approached the one of the UK. Along with an increase in the R\&D budget, additional direct funding was allocated for a group of leading Russian universities against federal projects. Today, leading Russian universities comprise: Lomonosov Moscow State University, St. Petersburg State University, 10 Federal Universities, 29 National Research Universities and 21 universities of the 5/100 project that aims at improving position of Russian universities in world university 
rankings. In response to an increase in funding, research output has been growing slowly but steadily.

Within Federal Target Programs substantial budgetary funds were allocated to subscribe most important scholarly resources for Russian academics and research institutes. The System of Informational Support of Science, Education, and Culture in Russia (System of Information Support) was established at the very end of 2005 and since that time acquisition of scholarly resources has closely followed research development that was reported in 2007 [16], 2011 [17$18]$ and 2016 [19-20].

Specifics of Russian national system of information support is in dual funding of subscription: with the state money allocated within federal projects and programs, and the money paid by institutions from their own budgets.

Institution budgets. Till 2014 the level of funding of Russian academics and research institutes was gradually growing due to direct funding. This resulted in a positive trend in library subscription, one could see that more and more money was spent on scholarly resources. Annual surveys conducted by NEICON since 2006 indicated that e-resource acquisition budgets of leading universities increased by 10 times from 2006 to 2016 . However, in 2014 due to economic crisis in Russia and almost a $100 \%$ drop in purchasing power of national currency, situation changed dramatically. The 2016 and 2017 surveys showed that research and educational institutions in Russia did not have enough money to maintain information support at appropriate level [21]. In result, the number of resources subscribed with institution budgets shrunk by $38 \%$ and $80 \%$ in 2015 and 2016, respectively.

State funding. Upon lasting impact of economic crisis, federal projects and programs make the only reliable sources of information support of Russian education and science. In this paper we confine our studies to national subscription projects funded by Ministry of Education and Science of Russian Federation (MES) and Russian Foundation for Basic Research (RFBR). In 
2017, resources subscribed within MES and RFBR projects comprise 18 full-text journals packages provided by world leading publishers focused at STM research areas. During the whole time span of MES projects, resources have been thoroughly selected by the experts of NEICON and leading Russian libraries in result of analysis performed in Web of Science Core Collection (WoS CC) with keyword search queries built for five above-mentioned priority areas. The selection process consists of four steps described in $[14,18]$ : 1. Preparation and refining of search query to search for the documents relevant to a priority area (for instance, Life Sciences); 2. Search of documents in WoS CC; 3. Preparation of ranked lists of the most productive periodicals in each priority area; 4. Identification of information provider that offers those periodicals. Journal packages of world publishers that contained the largest number of articles in priority areas were subscribed.

During all years the list of resources and number of subscribers could be corrected and changed after analysis of usage statistics, cost-efficiency and ROI. Last two years, MES also takes into account resource rankings built on results of library surveys.

In [14] we reported results of assessment and quantitative estimates of the level of information support provided by MES an RFBR projects: For each priority area we calculated parameter of information support P: proportion (percentage) of articles available in subscribed resources to world research output. $\mathrm{P}_{\mathrm{i}}=\mathrm{N}_{\mathrm{i}} / \mathrm{N}_{\mathrm{i}}$, where $\mathrm{N}_{\mathrm{i}}-$ number of articles in $i$ research area available via national subscription, $\mathrm{N}_{\mathrm{i} 0}-$ total number of articles in $i$ research area. Results obtained in five priority areas were as follows: Life Sciences $-74 \%$, Industry of Nanosystems 77\%; ITC Systems - 76\%; Environmental Management - 78\%; Energy Efficiency, Energy Saving and Nuclear Energy - 90\%. This proves a proper selection of resources in national subscription that provides a very high level of information support in the priority areas.

To fit one of the world standard classifications we extended our analysis to 22 research areas of Essential Science Indicators database. In result we got mapping of each of 18 full-text 
resources in the number of articles, proportion of citations and highly cited papers and parameter of information support.

\section{World experience in subscription and access to scientific information}

\subsection{Principles of formation of subscription in certain research organization}

Informational support is one of the main components of research activity and the role of library is to provide necessary information with maximal efficiency. This task is quite complicated even for research organizations with limited research interests because of limited budgets being almost impossible for multidisciplinary university libraries. Situation worsens within the crisis periods: either the 2008 Global economic crisis [21,22] or Russian economic crisis of 2014 [23].

The main principles for formation of subscription are its correspondence to research areas related to the research and educational needs and high quality of resources both in content and services. There are different approaches for selection of resources to subscribe: from expert evaluation to complex bibliometric methods and their combination with expert opinion and altmetrics.

For example, journal citation analysis, journal title and core list analysis, Electronic resource analysis and OCLC's WorldShare CE tool were used to evaluate the Interdisciplinary collection and further collection development at the College of Staten Island Library [24]. WorldShare CE was also used for mapping Conspectus subject divisions and categories to relevant university departments and evaluation holdings in comparison with department metrics to provide a fuller picture for collection development decision-making [25].

A new sophisticated model for the formation of ranked lists of scientific journals for library subscription based on bibliometric analysis was proposed in [26-28]. The model is based on the search in the abstract database of a set of articles that are semantically equivalent to a set of 
articles of the user or a group of users for which the repertoire of scientific periodicals is selected. In other words, the result of the request should be a collection of articles of the same thematic focus, which is expressed in the articles of the author himself. To achieve this goal, the authors used KeyWords Plus from the articles of employees of three scientific organizations in various scientific fields - biomedical, geological and physical-mathematical. KeyWords Plus, which is a brief retelling of each of the articles, is combined in the query into groups, the number of which, respectively, equaled the number of articles of employees of these organizations. As a result of the request, lists of articles were obtained exactly with the same sequences of keywords, which proved their semantic affinity. With the use of various filters, groups of journals are analyzed that concentrate the articles of interest to the greatest extent. The lists obtained are compared with the lists of journals most cited by the authors of the organizations under consideration, a considerable similarity of the lists in the field of natural sciences and a smaller one in the field of exact ones.

The last described method is quite good for choosing journals for monodisciplinary libraries. The selection of resources for multidisciplinary universities, consortium or national subscriptions must satisfy the following conditions: meeting the information needs of the maximum possible number of subscribers, as wide as possible range of scientific fields with efficient use of resources.

\subsection{Consortium subscription}

State funded projects in Russia use consortium licenses signed by a project operator on behalf of all member-institutions. Number of consortia subscribers is different for different resources and years and have varied from two dozens to several hundred for full-text journal databases. Noteworthy is that in 2017 MES project has subscribed 1600 Russian non-commercial institutions to WoS CC. This fact grounds the choice of WoS platform for analysis performed in our study. WoS CC is the database subscribed at a true national level in Russia. 
Following the widely known and well-reported international practices of library networking [29], collaborative collection development and consortia purchasing [30,31] with well established cost-effectiveness and return on investment [32-33], Russian consortia subscribe e-resources within "big deal" model when standard publisher packages of hundreds and thousands of titles are subscribed for all members of a consortium.

Big deal licenses have been negotiated by Russian national library consortium NEICON [17-18, 34] and were operated by NEICON (from 2006 to 2014) or Russian National Public Library for Science and Technology (since 2014 up to now).

Due to thorough market analysis, careful and intense negotiations with publishers, "big consortium deals" in Russia allow libraries to spend up to ten times less money compared to subscription to separate titles or selection of journals from standard packages.

After resources are subscribed, operators of MES project have monitored usage statistics, estimated cost efficiency, updated, and optimized the list of subscribed resources, number of site licenses and lists of subscribers. After several years of monitoring and optimization, cost-perdownload for each of 16 resources of "ministry" subscription usually reaches reasonable values of 2-5 USD/download ${ }^{1}$. If despite all efforts, cost-per-download remains high, subscription is canceled.

One has to say that during ten years of ministry subscription, only 3 full-text resources have been canceled.

\section{Methods}

1 We have perfomed our own investigation and asked international consortia and universities - members of ICOLC and publisher advisory boards to share with us criteria of appropriate price per dowmload values referred to consortium subscriptions and got 25 responses with values varying from 0.6 to 14 USD which we cannot publish for the reason of confidentiality. 
To analyze the compliance of the list of resources to be signed with the needs of Russian science we calculated the proportion of papers in subscribed resources in the overall number of documents indexed in WoS CC in last 5 years (2012-2016) together with their citation and share of documents in $10 \%$ of highly cited documents. The analysis was performed in 22 research areas used in Essential Science Indicators (ESI), so humanities appeared to be eliminated from our investigation.

We used InCites analytical instrument from Clarivate Analytics to get all the data on publications and citations. InCites accumulates publications from WoS CC (except Emerging Sources Citation Index) and permits one to obtain sophisticated bibliometric indicators for various publication sets, using either predefined filters (researchers, organizations, countries, journals, publishers, etc.), or custom data sets, exported from WoS CC. For our investigation we used filter for publishers according to the lists of imprints (Table 1).

Table 1. Publishers' imprints and journals for analysis in InCites

\begin{tabular}{|l|l|}
\hline Publisher & Imprints \\
\hline Springer-Nature & ADIS INT LTD, ASPEN LAW \& BUSINESS DIV ASPEN PUBLISHERS \\
& INC, BIOMED CENTRAL LTD, BIRKHAUSER VERLAG AG, \\
& SPRINGER BIRKHAUSER, CURRENT SCIENCE LTD, SPRINGER \\
& VIEWEG-SPRINGER FACHMEDIEN WIESBADEN GMBH, VIEWEG, \\
& FRIEDRICH VIEWEG \& SOHN VERLAGSGESELLSCHAFT MBH, \\
& GABLER VERLAG, GABLER VERLAG/SPRINGER FACHMEDIEN, \\
& HUMANA PRESS INC, KLUWER ACADEMIC PUBLISHERS, KLUWER \\
& ACADEMIC-HUMAN SCIENCES PRESS, KLUWER \\
& ACADEMIC/PLENUM PUBL, KLUWER ACADEMIC/PLENUM \\
& PUBLISHERS, KLUWER LAW INT, KLUWER LAW \\
& INTERNATIONAL, MACMILLAN INDIA LTD, MACMILLAN PRESS \\
& LTD, GAMSBERG MACMILLAN PUBLISHERS (PTY) LTD, \\
& PALGRAVE MACMILLAN LTD, NATURE PUBLISHING GROUP, \\
& PLENUM PRESS DIV PLENUM PUBLISHING CORP, \\
& SPRINGER/PLENUM PUBLISHERS, SPRINGER, SPRINGER BASEL \\
& AG, SPRINGER FRANCE, SPRINGER HEIDELBERG, SPRINGER \\
& IBERICA, SPRINGER INDIA, SPRINGER INT PUBLISHING AG, \\
& SPRINGER INTERNATIONAL PUBLISHING AG, SPRINGER JAPAN, \\
& SPRINGER JAPAN KK, SPRINGER LONDON LTD, SPRINGER \\
& PUBLISHING CO, SPRINGER SINGAPORE PTE LTD, SPRINGER \\
& SPEKTRUM/SPRINGER FACHMEDIEN, SPRINGER VERLAG PARIS, \\
\hline
\end{tabular}




\begin{tabular}{|c|c|}
\hline & $\begin{array}{l}\text { SPRINGER VS/SPRINGER FACHMEDIEN, SPRINGER WIEN, } \\
\text { SPRINGER-V D I VERLAG GMBH \& CO KG, SPRINGER-VERLAG } \\
\text { BERLIN, SPRINGER-VERLAG FRANCE, SPRINGER-VERLAG } \\
\text { ITALIA, SPRINGER-VERLAG ITALIA SRL, SPRINGER-VERLAG } \\
\text { LONDON LTD, SPRINGER-VERLAG SINGAPORE PTE LTD, } \\
\text { SPRINGER-VERLAG TOKYO, SPRINGER-VERLAG WIEN, } \\
\text { CONSULTANTS BUREAU/SPRINGER, COPERNICUS AN IMPRINT OF } \\
\text { SPRINGER, NY, MAIK NAUKA/INTERPERIODICA/SPRINGER, } \\
\text { SPEKTRUM AKADEMISCHER VERLAG-SPRINGER-VERLAG GMBH, } \\
\text { VIEWEG-TEUBNER VERLAG GMBH }\end{array}$ \\
\hline Elsevier & $\begin{array}{l}\text { ACADEMIC PRESS, ACADEMIC PRESS AUST, ACADEMIC PRESS } \\
\text { INC ELSEVIER SCIENCE, ACADEMIC PRESS LTD- ELSEVIER } \\
\text { SCIENCE LTD, ACADEMIC PRESS LTD-ELSEVIER SCIENCE LTD, } \\
\text { BAILLIERE TINDALL, ELSEVIER BUTTERWORTH-HEINEMANN, } \\
\text { BUTTERWORTH-HEINEMANN LTD, BUTTERWORTH-HEINEMANN } \\
\text { AUSTRALIA, BUTTERWORTH-HEINEMANN, CELL PRESS, } \\
\text { CHURCHILL LIVINGSTONE, CHURCHILL LIVINGSTONE INC } \\
\text { MEDICAL PUBLISHERS, CHURCHILL LIVINGSTONE LTD, } \\
\text { ELSEVIER DOYMA SL, EDICIONES DOYMA, EDICIONES DOYMA S } \\
\text { A, ELSEVIER, ELSEVIER ACADEMIC PRESS INC, ELSEVIER } \\
\text { ADVANCED TECHNOLOGY, ELSEVIER APPL SCI PUBL LTD, } \\
\text { ELSEVIER BRAZIL, ELSEVIER ESPANA S I, ELSEVIER FRANCE- } \\
\text { EDITIONS SCIENTIFIQUES MEDICALES ELSEVIER, ELSEVIER } \\
\text { GMBH, URBAN \& FISCHER VERLAG, ELSEVIER INC, ELSEVIER } \\
\text { INFORMACION PROFESSIONAL, S A, ELSEVIER MASSON, } \\
\text { ELSEVIER MASSON SAS EDITEUR, ELSEVIER IRELAND LTD, } \\
\text { ELSEVIER NORTH HOLLAND, ELSEVIER SCI LTD, ELSEVIER } \\
\text { SCIENCE BV, ELSEVIER SCIENCE INC, ELSEVIER SCIENCE } \\
\text { LONDON, ELSEVIER SCIENCE LTD, ELSEVIER SCIENCE } \\
\text { PUBLISHERS BV BIOMEDICAL DIVISION, ELSEVIER SCIENCE SA, } \\
\text { ELSEVIER SCIENTIFIC PUBL CO, ELSEVIER SCIENTIFIC } \\
\text { PUBLISHERS IRELAND LTD, ELSEVIER SINGAPORE PTE LTD, } \\
\text { ELSEVIER TAIWAN, ELSEVIER URBAN \& PARTNER SP Z O O, } \\
\text { ELSEVIER-IRCS LTD, ELSEVIER/NORTH-HOLLAND, JAI-ELSEVIER } \\
\text { LTD, JAI-ELSEVIER SCI BV, JAI-ELSEVIER SCIENCE INC, MOSBY- } \\
\text { ELSEVIER, HANLEY \& BELFUS-ELSEVIER INC, NORTH HOLLAND, } \\
\text { ELSEVIER SCIENCE PUBL BV, PERGAMON-ELSEVIER SCIENCE } \\
\text { LTD, W B SAUNDERS CO-ELSEVIER INC, EXCERPTA MEDICA INC- } \\
\text { ELSEVIER SCIENCE INC, GAUTHIER-VILLARS/EDITIONS } \\
\text { ELSEVIER, EDITIONS SCIENTIFIQUES ET MEDICALES ELSEVIER, } \\
\text { HANLEY \& BELFUS INC MED PUBLISHERS, JAI PRESS INC, } \\
\text { MASSON EDITEUR, MASSON INT NURSING PUBLICATIONS, } \\
\text { MASSON PUBL USA INC, MORGAN KAUFMANN PUB INC, MOSBY, } \\
\text { INC, PERGAMON PRESS, PERGAMON PRESS CANADA, PERGAMON } \\
\text { PRESS LTD, REED BUSINESS INFORMATION, REED BUSINESS } \\
\text { INFORMATION LTD, REED BUSINESS INFORMATION US, REED } \\
\text { HEALTHCARE COMMUNICATIONS, W B SAUNDERS CO LTD, } \\
\text { URBAN \& FISCHER VERLAG }\end{array}$ \\
\hline
\end{tabular}




\begin{tabular}{|c|c|}
\hline $\begin{array}{l}\text { TAYLOR \& } \\
\text { FRANCIS }\end{array}$ & $\begin{array}{l}\text { TAYLOR \& FRANCIS AS, TAYLOR \& FRANCIS AUSTRALASIA, } \\
\text { TAYLOR \& FRANCIS INC, TAYLOR \& FRANCIS LTD, CRC PRESS- } \\
\text { TAYLOR \& FRANCIS GROUP, ERLBAUM (UK) TAYLOR \& FRANCIS } \\
\text { PUBLISHERS, A A BALKEMA - TAYLOR \& FRANCIS THE } \\
\text { NETHERLANDS, GARLAND SCIENCE, TAYLOR \& FRANCIS, } \\
\text { CARFAX PUBLISHING-TAYLOR \& FRANCIS GROUP, ROUTLEDGE } \\
\text { JOURNALS, TAYLOR \& FRANCIS LTD, AUERBACH } \\
\text { PUBLICATIONS, TAYLOR \& FRANCIS GROUP, HARWOOD ACAD } \\
\text { PUBL GMBH, TAYLOR \& FRANCIS GROUP, GORDON BREACH } \\
\text { PUBLISHING, TAYLOR \& FRANCIS GROUP, LAWRENCE ERLBAUM } \\
\text { ASSOC INC-TAYLOR \& FRANCIS, ROUTLEDGE, MANEY } \\
\text { PUBLISHING, HAWORTH PRESS INC, HAWTHORN PRESS, MARCEL } \\
\text { DEKKER, MARCEL DEKKER INC, LAWRENCE ERLBAUM ASSOC } \\
\text { LTD, LAWRENCE ERLBAUM ASSOC LTD PUBL, LAWRENCE } \\
\text { ERLBAUM ASSOC PUBL, FRANK CASS AND CO LTD, FRANK CASS } \\
\text { CO LTD, LANDES BIOSCIENCE, HELDREF PUBLICATIONS, M E } \\
\text { SHARPE INC, INFORMA HEALTHCARE, GORDON AND BREACH } \\
\text { SCIENCE PUBL, CHAPMAN \& HALL, CHAPMAN \& HALL INC, } \\
\text { CHAPMAN \& HALL/CRC PRESS, CHAPMAN HALL LTD, VILNIUS } \\
\text { GEDIMINAS TECH UNIV, VILNIUS GEDIMINAS TECHNICAL UNIV } \\
\text { PRESS, TECHNIKA, HAWORTH HOSPITALITY PRESS, HAWORTH } \\
\text { MEDICAL PRESS, CRC PRESS LLC }\end{array}$ \\
\hline WILEY & $\begin{array}{l}\text { WILEY, WILEY CHANCERY, WILEY EASTERN LTD, WILEY } \\
\text { PERIODICALS, WILEY PERIODICALS, INC, WILEY-BLACKWELL, } \\
\text { WILEY-LISS, WILEY-LISS, INC, WILEY-V C H VERLAG GMBH, } \\
\text { WILEY-VCH, INC, JOHN WILEY \& SONS, JOHN WILEY \& SONS INC, } \\
\text { JOHN WILEY \& SONS LTD, SCRIPTA TECHNICA-JOHN WILEY \& } \\
\text { SONS, JOSSEY-BASS INC PUBL-JOHN WILEY \& SONS, AMER } \\
\text { GEOPHYSICAL UNION, MUNKSGAARD, BLACKWELL } \\
\text { MUNKSGAARD, BLACKWELL PUBL, BLACKWELL PUBL LTD, } \\
\text { BLACKWELL PUBLISHERS, BLACKWELL PUBLISHING ASIA, } \\
\text { BLACKWELL PUBLISHING INC, BLACKWELL SCIENCE INC, } \\
\text { BLACKWELL SCIENCE PUBL, BLACKWELL VERLAG GMBH, } \\
\text { BLACKWELL WISSENSCHAFTS-VERLAG GMBH, ARNETTE } \\
\text { BLACKWELL S A }\end{array}$ \\
\hline SAGE & $\begin{array}{l}\text { SAGE PUBLICATIONS INC, SAGE PUBLICATIONS INDIA PVT LTD, } \\
\text { SAGE PUBLICATIONS INDIA PVT LTD, SAGE PUBLICATIONS LTD }\end{array}$ \\
\hline $\begin{array}{l}\text { OXFORD } \\
\text { UNIVERSITY } \\
\text { PRESS }\end{array}$ & $\begin{array}{l}\text { OXFORD UNIV PRESS, OXFORD UNIV PRESS INC, OXFORD UNIV } \\
\text { PRESS CAPE TOWN, OXFORD UNIV PRESS DELHI }\end{array}$ \\
\hline $\begin{array}{l}\text { CAMBRIDGE } \\
\text { UNIVERSITY } \\
\text { PRESS }\end{array}$ & $\begin{array}{l}\text { CAMBRIDGE UNIV PRESS, CAMBRIDGE INTERNATIONAL } \\
\text { SCIENCE PUBLISHING }\end{array}$ \\
\hline IEEE & $\begin{array}{l}\text { INSTITUTE OF ELECTRICAL AND ELECTRONICS ENGINEERS, } \\
\text { INSTITUTE OF ELECTRICAL AND ELECTRONICS ENGINEERS INC., }\end{array}$ \\
\hline
\end{tabular}




\begin{tabular}{|l|l|}
\hline & $\begin{array}{l}\text { IEEE COMPUTER SOCIETY, IEEE ADVANCING TECHNOLOGY FOR } \\
\text { HUMANITY, IEEE SYSTEMS, MAN, AND CYBERNETICS SOCIETY, } \\
\text { IEEE EDUCATION SOCIETY, INSTITUTE OF ELECTRICAL AND } \\
\text { ELECTRONIC ENGINEERS CANADA, INSTITUTE OF ELECTRICAL } \\
\text { AND ELECTRONICS ENGINEERS, IEEE TECHNICAL COMMITTEE } \\
\text { ON LEARNING TECHNOLOGY, IEEE SOLID-STATE CIRCUITS } \\
\text { SOCIETY, IEEE POWER ELECTRONICS SOCIETY, IEEE } \\
\text { GEOSCIENCE AND REMOTE SENSING SOCIETY, IEEE ELECTRON } \\
\text { DEVICES SOCIETY, IEEE ELECTROMAGNETIC COMPATIBILITY } \\
\text { SOCIETY, IEEE CONTROL SYSTEMS SOCIETY, IEEE } \\
\text { COMPUTATIONAL INTELLIGENCE SOCIETY, IEEE } \\
\text { COMMUNICATIONS SOCIETY, IEEE CIRCUITS AND SYSTEMS } \\
\text { SOCIETY, ALASKA NURSES ASSOCIATION }\end{array}$ \\
\hline THIEME & $\begin{array}{l}\text { ONLY 5 TITLES ARE CURRENTLY SUBSCRIBED WITHIN } \\
\text { "MINISTRY PROJECT": PHARMACOPSYCHIATRY, PLANTA } \\
\text { MEDICA, SYNFACTS, SYNLETT, AND SYNTHESIS }\end{array}$ \\
\hline $\begin{array}{l}\text { OPTICAL } \\
\text { SOCIETY OF } \\
\text { AMERICA }\end{array}$ & OPTICAL SOCIETY OF AMERICA \\
\hline $\begin{array}{l}\text { ROYAL } \\
\text { SOCIETY OF } \\
\text { CHEMISTRY }\end{array}$ & ROYAL SOCIETY OF CHEMISTRY \\
\hline $\begin{array}{l}\text { AMERICAN } \\
\text { CHEMICAL } \\
\text { SOCIETY }\end{array}$ & AMERICAN CHEMICAL SOCIETY \\
\hline $\begin{array}{l}\text { AMERICAN } \\
\text { INSTITUTE OF } \\
\text { PHYSICS }\end{array}$ & $\begin{array}{l}\text { AIP PUBLISHING LLC, AMERICAN INSTITUTE OF PHYSICS, } \\
\text { AMERICAN INSTITUTE OF PHYSICS INC., AMERICAN INSTITUTE } \\
\text { OF PHYSICS PUBLISHING LLC }\end{array}$ \\
\hline $\begin{array}{l}\text { AMERICAN } \\
\text { PHYSICAL } \\
\text { SOCIETY }\end{array}$ & AMERICAN PHYSICAL SOCIETY \\
\hline SPIE & $\begin{array}{l}\text { SPIE-INT SOC OPTICAL ENGINEERING, SPIE-SOC PHOTO-OPTICAL } \\
\text { INSTRUMENTATION ENGINEERS, IS\&T \& SPIE }\end{array}$ \\
\hline
\end{tabular}

As to our knowledge, this is the first time when analytical possibilities of InCites has been used for collection development and quantitative and qualitative evaluation of the level of information support of scholarly research.

Operators of RFBR project provided the data on the Elsevier and Springer-Nature usage statistics (full-text downloads in 2016). The usage data of MES project is available on the 
webpage of Russian National Public Library for Science and Technology ${ }^{2}$. The following resources are considered as MES or "ministry subscription":

${ }^{2}$ URL: http://konkurs.vlibrary.ru/?id=KonkursStaticStat 
- AAAS

- $\mathrm{ACS}$

- AIP

- APS
- CUP

- IEEE

- IOP

- NPG
- OSA

- OUP

- RSC

- SAGE
- SPIE

- $\mathrm{T} \& \mathrm{~F}$

- Thieme

- Wiley

"RFBR subscription" includes Springer - Nature journal package and Freedom Collection of Elsevier.

\section{Results}

\subsection{Analysis of publication output in Russia in comparison with world output by ESI classificatory}

The publication distribution of Russian universities and research organizations in WoS CC over ESI research areas differs essentially from that of the world total (Fig.1).
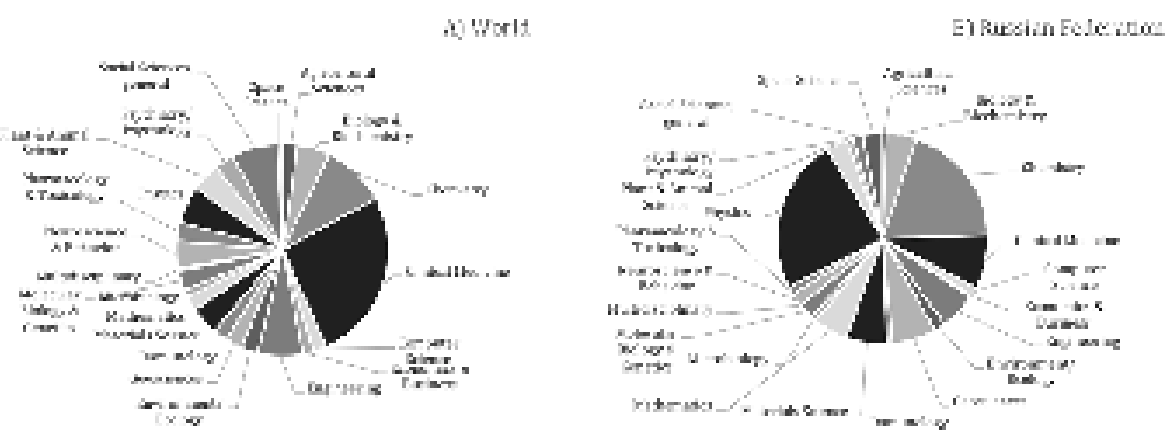

Fig.1. World (A) and Russian Federation (B) output in Web of Science Core Collection (20122016) by ESI research areas

A quarter of world publications are in Clinical medicine, while in Russia a half of publications are in Physics and Chemistry, Clinical medicine being only in the third place by number of publications. Such areas as Economics \& Business, Immunology, Psychiatry/Psychology, Social Sciences are presented in Russian publications in WoS CC less than in world average, while Space Sciences, Geosciences and Materials Sciences occupy the larger share in total publication output than in world average. 
Only leading multidisciplinary universities such as Moscow State University or Saint Petersburg State University ( $\mathrm{SPbSU}$ ) demonstrate almost the same distribution of publications as Russian average, other universities and research organizations have different distribution of research interests with prevalence of one or several research areas (Table 2).

Table 2. Research diversity in some Russian Universities as to compare with whole Russian Federation output and World output (WoS CC, 2012-2016)

\begin{tabular}{|c|c|c|c|c|c|c|c|c|}
\hline & $\begin{array}{l}\overline{0} \\
\overline{0}\end{array}$ & 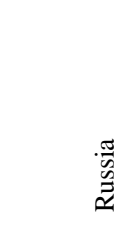 & 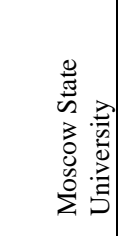 & 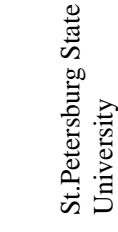 & 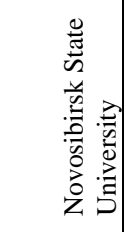 & 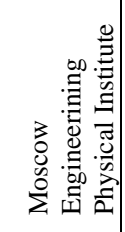 & 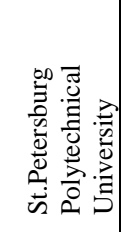 & 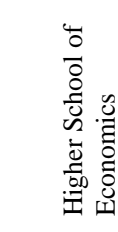 \\
\hline Physics & $5.7 \%$ & $23.6 \%$ & $27.6 \%$ & $21.5 \%$ & $34.0 \%$ & $67.1 \%$ & $52.7 \%$ & $14.6 \%$ \\
\hline Chemistry & $9.6 \%$ & $19.1 \%$ & $18.4 \%$ & $24.6 \%$ & $27.5 \%$ & $8.8 \%$ & $10.6 \%$ & $2.0 \%$ \\
\hline Clinical Medicine & $26.2 \%$ & $8.3 \%$ & $2.8 \%$ & $2.2 \%$ & $1.7 \%$ & $0.6 \%$ & $1.1 \%$ & $1.4 \%$ \\
\hline Geosciences & $2.5 \%$ & $6.7 \%$ & $5.1 \%$ & $7.8 \%$ & $8.5 \%$ & $0.7 \%$ & $0.8 \%$ & $1.2 \%$ \\
\hline Materials Science & $4.3 \%$ & $6.3 \%$ & $3.8 \%$ & $4.9 \%$ & $3.3 \%$ & $4.4 \%$ & $9.9 \%$ & $0.7 \%$ \\
\hline Engineering & $6.9 \%$ & $5.6 \%$ & $2.9 \%$ & $4.0 \%$ & $4.8 \%$ & $9.2 \%$ & $9.2 \%$ & $6.7 \%$ \\
\hline Mathematics & $2.2 \%$ & $5.2 \%$ & $8.6 \%$ & $7.4 \%$ & $7.7 \%$ & $3.2 \%$ & $1.1 \%$ & $25.5 \%$ \\
\hline Biology \& Biochemistry & $4.9 \%$ & $4.5 \%$ & $8.3 \%$ & $4.3 \%$ & $3.3 \%$ & $0.4 \%$ & $4.5 \%$ & $1.0 \%$ \\
\hline Plant \& Animal Science & $4.3 \%$ & $3.3 \%$ & $4.0 \%$ & $4.1 \%$ & $0.8 \%$ & $0.0 \%$ & $0.2 \%$ & $0.2 \%$ \\
\hline Space Science & $0.8 \%$ & $3.0 \%$ & $5.6 \%$ & $5.4 \%$ & $0.4 \%$ & $2.9 \%$ & $3.4 \%$ & $1.3 \%$ \\
\hline Molecular Biology \& Genetics & $3.0 \%$ & $2.3 \%$ & $3.1 \%$ & $3.4 \%$ & $3.5 \%$ & $0.1 \%$ & $1.7 \%$ & $0.5 \%$ \\
\hline Neuroscience \& Behavior & $4.0 \%$ & $2.0 \%$ & $1.1 \%$ & $1.4 \%$ & $0.7 \%$ & $0.1 \%$ & $0.7 \%$ & $4.2 \%$ \\
\hline Environment/Ecology & $2.6 \%$ & $1.7 \%$ & $1.4 \%$ & $1.2 \%$ & $0.2 \%$ & $0.1 \%$ & $0.2 \%$ & $0.3 \%$ \\
\hline Social Sciences, general & $7.0 \%$ & $1.6 \%$ & $0.7 \%$ & $1.5 \%$ & $0.3 \%$ & $0.2 \%$ & $0.2 \%$ & $20.0 \%$ \\
\hline Computer Science & $2.0 \%$ & $1.3 \%$ & $0.9 \%$ & $0.8 \%$ & $0.9 \%$ & $0.6 \%$ & $1.1 \%$ & $4.2 \%$ \\
\hline Pharmacology \& Toxicology & $2.8 \%$ & $1.2 \%$ & $0.7 \%$ & $0.6 \%$ & $0.5 \%$ & $0.1 \%$ & $0.4 \%$ & $0.5 \%$ \\
\hline Psychiatry/Psychology & $3.0 \%$ & $0.9 \%$ & $1.1 \%$ & $1.5 \%$ & $0.2 \%$ & $0.3 \%$ & $0.3 \%$ & $5.5 \%$ \\
\hline Microbiology & $1.1 \%$ & $0.9 \%$ & $1.1 \%$ & $1.2 \%$ & $0.6 \%$ & $0.0 \%$ & $0.5 \%$ & $0.0 \%$ \\
\hline Immunology & $2.1 \%$ & $0.8 \%$ & $0.2 \%$ & $0.3 \%$ & $0.3 \%$ & $0.0 \%$ & $0.2 \%$ & $0.1 \%$ \\
\hline Agricultural Sciences & $2.3 \%$ & $0.6 \%$ & $1.7 \%$ & $0.4 \%$ & $0.1 \%$ & $0.0 \%$ & $0.0 \%$ & $0.0 \%$ \\
\hline Economics \& Business & $1.6 \%$ & $0.3 \%$ & $0.1 \%$ & $0.6 \%$ & $0.1 \%$ & $0.0 \%$ & $0.1 \%$ & $8.9 \%$ \\
\hline Multidisciplinary & $0.4 \%$ & $0.1 \%$ & $0.1 \%$ & $0.1 \%$ & $0.0 \%$ & $0.0 \%$ & $0.0 \%$ & $0.2 \%$ \\
\hline
\end{tabular}

That is the reason of different approaches in formation of subscriptions to electronic resources on the level of separate organization or national level. The subscription in such universities as SPbSU can serve as model one for national subscription. On the other hand, we 
must take into account that lack of publications in several research areas may be the consequence of insufficient access to information resources.

\subsection{Coverage of ESI research areas by national subscription in number of publications, citations, and proportion of highly cited papers}

One of the main criteria for subscription is the completeness of publication presentation. We evaluated this criterion by number of publications in Web of Science Core Collection through ESI research areas. The information on the absolute number of publications in journals of subscribed publishers is given in Table 3 . 
Table 3. Absolute number of articles, published in journals in subscribed resources (WoS CC, 2012-2016)

\begin{tabular}{|c|c|c|c|c|c|c|c|c|c|c|c|c|c|c|c|c|c|c|c|}
\hline & & RFBR subc & ription & & & & & Mini & try of E & ducation & and $\mathrm{Sc}$ & ience sub & cription & & & & & & \\
\hline & 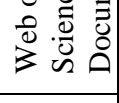 & $\begin{array}{r}\frac{0}{7} \\
0 \\
0 \\
\underline{\pi} \\
\end{array}$ & 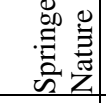 & $\frac{2}{3}$ & $\begin{array}{r}\frac{1}{2} \\
-1 \\
\end{array}$ & 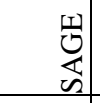 & 官 & 它 & 穿 & 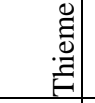 & $\frac{2}{4}$ & $\underbrace{2}_{4}$ & $\mathrm{z}$ & $\begin{array}{l}2 \\
\frac{1}{2}\end{array}$ & อి & $\begin{array}{l}\pi \\
0 \\
0\end{array}$ & $\begin{array}{l}U \\
\approx \\
\simeq\end{array}$ & 竞 & \\
\hline Agricultural Sciences & 221367 & 62782 & 22727 & 21941 & 11171 & 1346 & 388 & 5138 & & & 7 & 7024 & & & & & 1566 & & 87277 \\
\hline $\begin{array}{l}\text { Biology \& } \\
\text { Biochemistry }\end{array}$ & 480522 & 124700 & 60644 & 60812 & 17132 & 596 & 12965 & 66 & & & 2133 & 11285 & & & 1272 & 1715 & 8542 & & 178660 \\
\hline Chemistry & 940410 & 212989 & 88813 & 107067 & 24496 & 3105 & 1524 & & & 4973 & 563 & 211472 & 20381 & & 3426 & & 122797 & & 138804 \\
\hline Clinical Medicine & 2561321 & 551194 & 402351 & 379118 & 52530 & 55869 & 114276 & 3611 & & & 279 & & & & 727 & & & 2517 & 998849 \\
\hline Computer Science & 198106 & 59016 & 36598 & 5835 & 3399 & 3447 & 5358 & 676 & 12093 & & 15 & & & & & 722 & & & 70947 \\
\hline Economics \& Business & 152536 & 39333 & 21069 & 25217 & 16206 & 7249 & 5162 & 4035 & & & 101 & & & & & & & & 34164 \\
\hline Engineering & 670640 & 224473 & 77113 & 23397 & 40077 & 17786 & 321 & 5478 & 2661 & & 31 & 4580 & 1585 & & 5429 & 735 & & 4922 & 262052 \\
\hline Environment/ Ecology & 253782 & 56451 & 55551 & 39596 & 13665 & 781 & 585 & 1880 & & & 524 & 9047 & & & 1718 & & 1652 & & 72332 \\
\hline Geosciences & 243617 & 64965 & 39111 & 14447 & 8028 & 1015 & 3002 & 1986 & & & 597 & & & & 453 & & & 1034 & 108979 \\
\hline Immunology & 209768 & 56822 & 29205 & 29397 & 7207 & 1719 & 8198 & & & & 241 & & & & & & & & 76979 \\
\hline Materials Science & 421949 & 151794 & 58108 & 30406 & 15692 & 6219 & 5 & 4162 & & & 72 & 17758 & 7386 & & 10588 & 1437 & 27481 & & 90841 \\
\hline Mathematics & 216083 & 48652 & 46687 & 8072 & 12819 & 512 & 3307 & 5561 & & & 8 & & & & 792 & & & & 89673 \\
\hline Microbiology & 111994 & 18387 & 24807 & 6577 & 280 & & 2790 & 926 & & & 185 & & & & & & & & 58042 \\
\hline $\begin{array}{l}\text { Molecular Biology \& } \\
\text { Genetics }\end{array}$ & 295316 & 52995 & 65373 & 33888 & 10195 & & 7649 & 1015 & & & 2212 & & & & 2778 & & 1436 & & 117775 \\
\hline Multidisciplinary & 39585 & 10445 & 10161 & 127 & 146 & & 81 & & & & 6670 & & & & & & & & 11955 \\
\hline $\begin{array}{l}\text { Neuroscience \& } \\
\text { Behavior }\end{array}$ & 394612 & 111046 & 72900 & 60889 & 6500 & 17548 & 17709 & 3413 & & 293 & 478 & 845 & & & 706 & & & 131 & 102154 \\
\hline $\begin{array}{l}\text { Pharmacology \& } \\
\text { Toxicology }\end{array}$ & 274875 & 74326 & 33008 & 38294 & 26886 & 5732 & 4590 & & & 5406 & 26 & 5321 & & & & & 1977 & & 79309 \\
\hline Physics & 552952 & 91142 & 77593 & 8324 & 8053 & & 803 & 1056 & & & 713 & 6479 & 56644 & 94171 & 62650 & 35770 & 8950 & 670 & 99934 \\
\hline Plant \& Animal Science & 417843 & 62144 & 58204 & 57200 & 16652 & 2544 & 27420 & 4832 & & & 303 & & & & & & & & 188544 \\
\hline Psychiatry/ Psychology & 297138 & 56989 & 65398 & 40424 & 38998 & 27747 & 7378 & 6406 & & & 108 & & & & & & & & 53690 \\
\hline Social Sciences, general & 680643 & 95684 & 135233 & 78465 & 98498 & 76376 & 40858 & 34583 & 583 & & 186 & & & & & & 332 & & 119845 \\
\hline Space Science & 74541 & 6631 & 7372 & 766 & 162 & & 16209 & 475 & & & 306 & & & & 24329 & & & & 18291 \\
\hline
\end{tabular}


One can see that the world largest multidisciplinary publishers, such as Elsevier, SpringerNature, Wiley, Taylor \& Francis, et all provide access in all ESI research areas to more than a half of documents indexed in WoS CC.

However, resources of world leading learning societies are also of a great importance. ACS with its 48 titles provides $22.5 \%$ of WoS CC output in Chemistry being the world's second after Elsevier with $22.6 \%$. That is why, the list of subscribed resources contains not only multidisciplinary but also specialized packages that add in Russian national subscription lots of high-quality articles. Along with $22.5 \%$ of ACS, RSC provides $13 \%$ of world flow in Chemistry. In Physics APS provides $17.0 \%$ of world output, AIP $-10.2 \%$, IoP $-13.3 \%$, OSA $-6.5 \%$. The sum over those four learning societies gives $47 \%$ of WoS output in Physics - more than 33.5\% provided by Elsevier, Springer-Nature, Wiley, and T\&F. Besides IoP provides $32.6 \%$ of world output in Space Science, RSC - 6\% in Material Sciences, and IEEE - 6\% of Computer Science. These numbers indicate to a very important role of learning society publishers in information support that cannot be neglected. The analysis of all resources shows that in average the MES and RFBR subscriptions provide the access to about $70 \%$ of WoS CC content. The detailed information by ESI research areas is presented in Fig. 2.

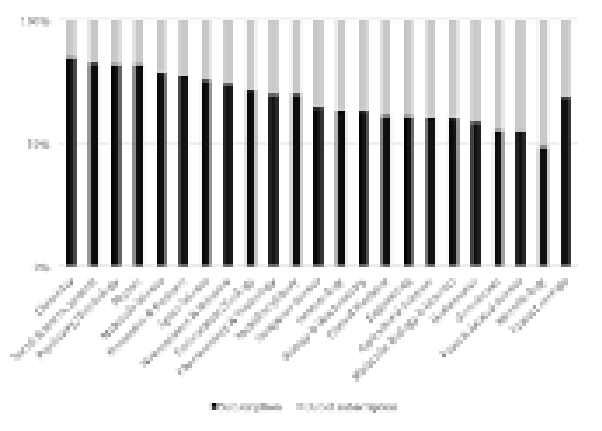

Fig.2. The proportion of articles in subscribed resources to world research output

The diagram in Fig. 2 shows that the best situation is with access to publications in Chemistry (about 90\%) and the worst - in Microbiology (less that 50\%). The input of RFBR and 
MES subscriptions is almost equal (Table 3), though RFBR provides access only to SpringerNature and Elsevier.

The quality of subscribed resources can be to some extent characterized by number of received citations (Fig.3).

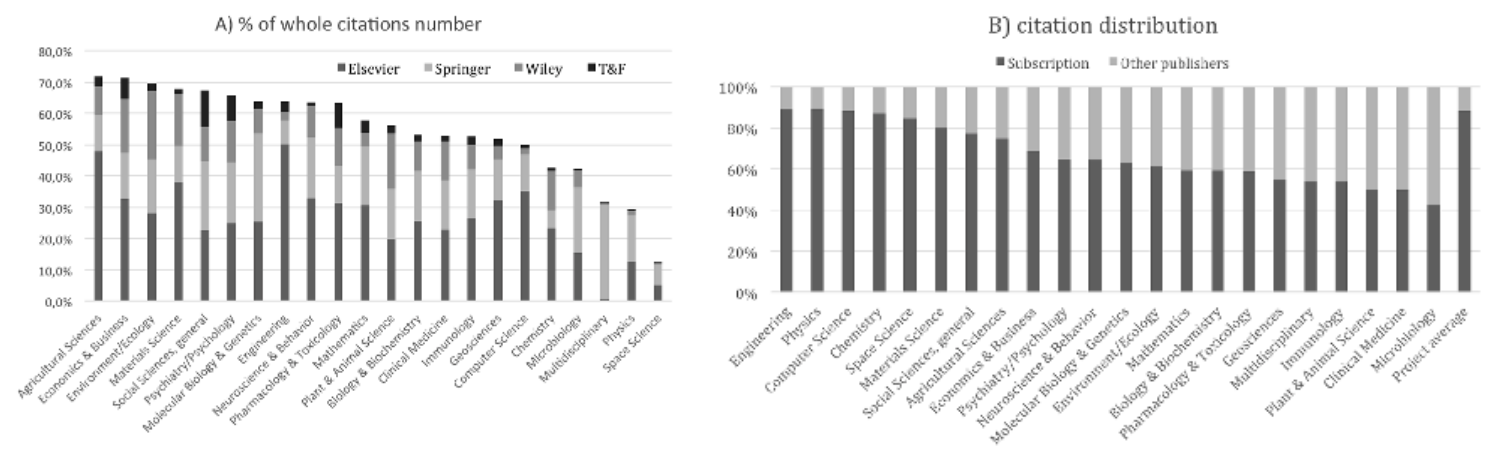

Fig. 3. The quality of subscribed resources by proportion of received citations (A - largest multidisciplinary publishers, B - Subscribed resources versus other publishers)

Fig. 3A shows that even multidisciplinary resources provide up to $70 \%$ of total citations in ESI research areas however, in Physics or Chemistry the learning society publishers such as APS or RSC play the most important role (compare Fig. 3A and Fig. 3B).

Even taking into account the strict rules for content selection in Web of Science Core Collection itself, one can see that subscribed resources present the highest-level journals. For example, more than $70 \%$ of journals in Elsevier Freedom collection (RFBR subscription) are in Q1 and Q2 by their impact-factor and only 11\% are in Q4. For certain research areas the situation may differ but in the whole the number of Q1 and Q2 journals in subscribed resources is higher than Q3 and Q4.

The number of citations by publishers (in percent of total citations) depends mainly on total number of publications, so more adequate indicator of quality is average citation per publication or such indicator as Category Normalized Citation Impact (CNCI). CNCI shows the relation of 
average citation in selected papers to world average in the same subject category taking into account the date of publication and its type (article, review, et al.). In Physics, for example, the total citation number is counted for APS journals, while the highest CNCI value is demonstrated by physical publications in AAAS and journals of ACS and RSC.

\subsection{Open access journals in subscribed resources}

The common trend of research organization now is open access to research information, including publications in scholarly journals. The number of OA journals is growing, many journals with traditional subscription models use the hybrid approach and publish some articles in open access for additional charges (Article Processing Charge, APC). The proportion of OA articles in WoS CC (only full OA journals) grew from $6.8 \%$ in 2012 up to $10.6 \%$ in 2016 , increasing the availability of full-texts to researchers. The proportion of OA journals in subscribed resources under consideration is much lower than for other publishers (Table 4) and differs greatly for certain ESI research areas. This data coincides with the data on different types of OA across publishers and disciplines in [35].

Table 4. The proportion of publications in open access journals in WoS CC

\begin{tabular}{|c|c|c|c|}
\hline & $\% \mathrm{OA}$ in Wos CC & $\begin{array}{c}\% \mathrm{OA} \text { in subscribed } \\
\text { publishers }\end{array}$ & $\begin{array}{l}\text { \%OA in other } \\
\text { publishers }\end{array}$ \\
\hline Microbiology & $29.3 \%$ & $7.8 \%$ & $21.5 \%$ \\
\hline Multidisciplinary & $24.7 \%$ & $0.1 \%$ & $24.5 \%$ \\
\hline $\begin{array}{l}\text { Molecular Biology \& } \\
\text { Genetics }\end{array}$ & $20.7 \%$ & $7.7 \%$ & $13.0 \%$ \\
\hline Immunology & $18.2 \%$ & $4.4 \%$ & $13.9 \%$ \\
\hline Biology \& Biochemistry & $14.4 \%$ & $4.0 \%$ & $10.4 \%$ \\
\hline Environment/Ecology & $13.0 \%$ & $2.8 \%$ & $10.2 \%$ \\
\hline Plant \& Animal Science & $12.6 \%$ & $2.3 \%$ & $10.4 \%$ \\
\hline Agricultural Sciences & $12.3 \%$ & $1.4 \%$ & $10.9 \%$ \\
\hline Geosciences & $12.0 \%$ & $0.9 \%$ & $11.2 \%$ \\
\hline Neuroscience \& Behavior & $11.7 \%$ & $2.2 \%$ & $9.5 \%$ \\
\hline Mathematics & $9.3 \%$ & $2.8 \%$ & $6.5 \%$ \\
\hline Clinical Medicine & $8.7 \%$ & $2.5 \%$ & $6.2 \%$ \\
\hline Physics & $8.5 \%$ & $4.9 \%$ & $3.5 \%$ \\
\hline Psychiatry/Psychology & $7.9 \%$ & $2.0 \%$ & $5.9 \%$ \\
\hline
\end{tabular}




\begin{tabular}{|l|r|r|r|}
\hline Social Sciences, general & $6.8 \%$ & $2.8 \%$ & $4.0 \%$ \\
\hline Computer Science & $6.2 \%$ & $3.9 \%$ & $2.3 \%$ \\
\hline Materials Science & $6.2 \%$ & $1.4 \%$ & $4.8 \%$ \\
\hline Pharmacology \& Toxicology & $5.9 \%$ & $0.7 \%$ & $5.1 \%$ \\
\hline Engineering & $5.6 \%$ & $1.1 \%$ & $4.5 \%$ \\
\hline Chemistry & $4.9 \%$ & $0.3 \%$ & $4.6 \%$ \\
\hline Economics \& Business & $2.4 \%$ & $0.2 \%$ & $2.3 \%$ \\
\hline Space Science & $1.3 \%$ & $0.0 \%$ & $1.3 \%$ \\
\hline
\end{tabular}

The proportion of OA journals for different research areas is to be taken into account while selecting the resources for subscription. For example, the highest level of OA for Microbiology compensates the low availability of journals on microbiology in subscribed resources. The negative correlation of OA (percent of total publications in ESI subject area) and availability of subscribed journals is demonstrated in Fig. 4.

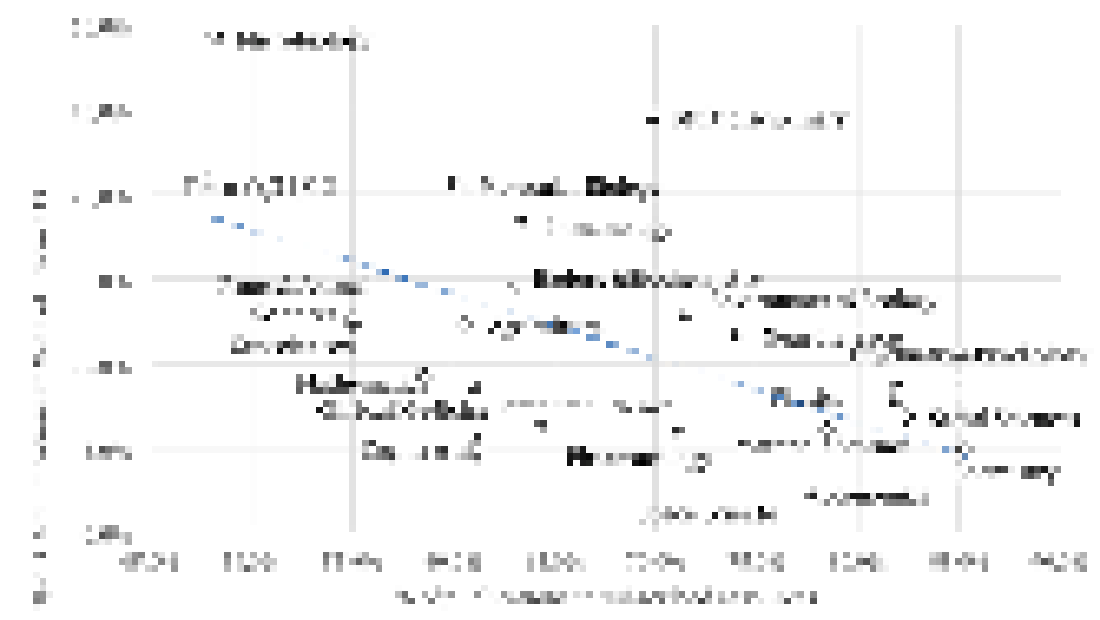

Fig.4. Proportion of OA publications and share of research output provided by subscribed journals

We obtained the availability of full-texts with taking into account the OA journals (Fig. 5). 


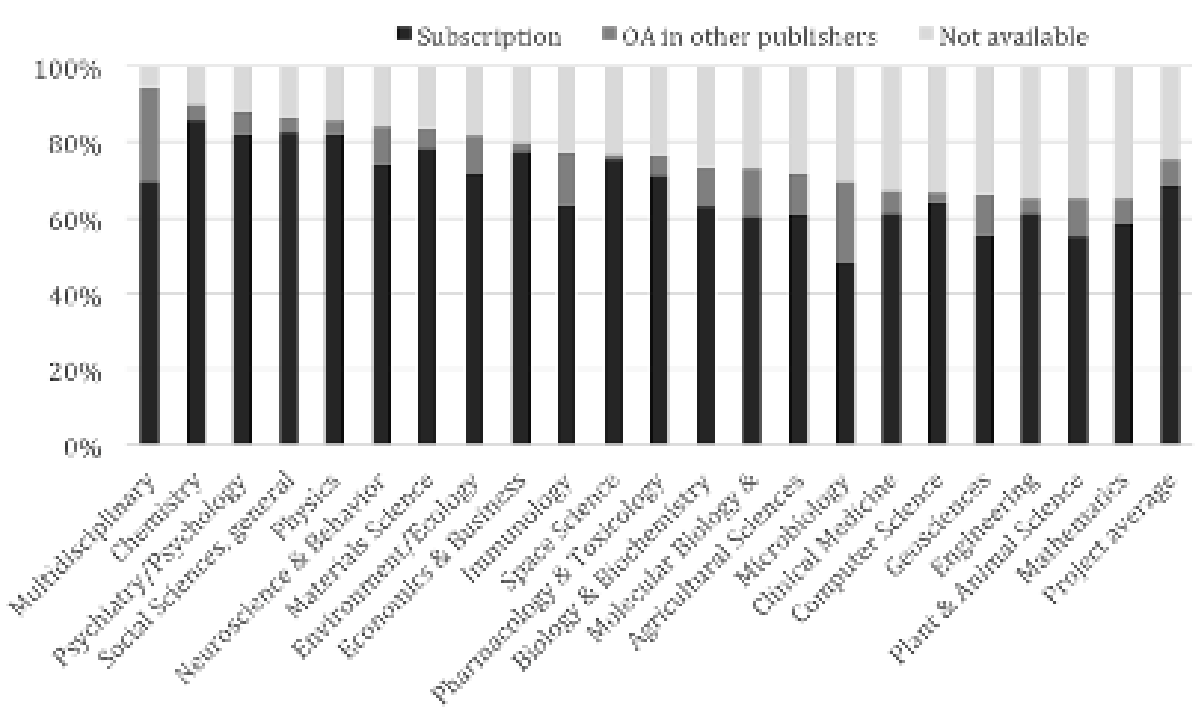

Fig.5. Availability of publications in WoS CC by ESI research areas (per cents of total number in category)

One can see that real proportion increases from $68 \%$ average for subscribed resources (Fig. 2) to $75 \%$. The low level of availability in Microbiology, Plant \& Animal Science and Geosciences (about or less than 50\%) is compensated with relatively high proportion of OA journals.

\subsection{Russian publications in subscribed journals}

One of the principles for resource selection for subscription is their usability not only as sources of information but also as channels of scientific communication, namely, publication of research results. We analyzed distribution of Russian publications in journals of different publishers and discovered that more than a half are published in the journals presented in Springer-Nature and Freedom Collection of Elsevier, i.e. subscribed by RFBR.

About $82 \%$ of Russian publications are in journals subscribed in the frame of MES and RFBR subscription. Almost all Russian publications in chemistry, physics and other natural sciences are in subscribed journals (Table 5).

Table 5. Total distribution of Russian publications by journals availability 


\begin{tabular}{|c|c|c|c|c|c|}
\hline & \multicolumn{3}{|c|}{ Total number } & \multicolumn{2}{|c|}{$\%$ of total } \\
\hline & $\begin{array}{c}\text { Web of } \\
\text { Science } \\
\text { Documents }\end{array}$ & $\begin{array}{l}\text { Subscribed } \\
\text { publishers }\end{array}$ & $\begin{array}{c}\text { Other } \\
\text { Publishers }\end{array}$ & Subscription & $\begin{array}{l}\text { Other } \\
\text { publishers }\end{array}$ \\
\hline Chemistry & 33530 & 31617 & 1913 & $94.3 \%$ & $5.7 \%$ \\
\hline Agricultural Sciences & 1128 & 1033 & 95 & $91.6 \%$ & $8.4 \%$ \\
\hline Engineering & 9883 & 8870 & 1013 & $89.8 \%$ & $10.2 \%$ \\
\hline Physics & 41534 & 36809 & 4725 & $88.6 \%$ & $11.4 \%$ \\
\hline Computer Science & 2261 & 1995 & 266 & $88.2 \%$ & $11.8 \%$ \\
\hline Environment/Ecology & 2959 & 2577 & 382 & $87.1 \%$ & $12.9 \%$ \\
\hline Materials Science & 11143 & 9569 & 1574 & $85.9 \%$ & $14.1 \%$ \\
\hline Biology \& Biochemistry & 7929 & 6710 & 1219 & $84.6 \%$ & $15.4 \%$ \\
\hline Geosciences & 11743 & 9638 & 2105 & $82.1 \%$ & $17.9 \%$ \\
\hline Economics \& Business & 522 & 418 & 104 & $80.1 \%$ & $19.9 \%$ \\
\hline Space Science & 5266 & 4160 & 1106 & $79.0 \%$ & $21.0 \%$ \\
\hline $\begin{array}{l}\text { Pharmacology \& } \\
\text { Toxicology }\end{array}$ & 2063 & 1627 & 436 & $78.9 \%$ & $21.1 \%$ \\
\hline Immunology & 1349 & 1045 & 304 & $77.5 \%$ & $22.5 \%$ \\
\hline Mathematics & 9060 & 6739 & 2321 & $74.4 \%$ & $25.6 \%$ \\
\hline Psychiatry/Psychology & 1653 & 1189 & 464 & $71.9 \%$ & $28.1 \%$ \\
\hline $\begin{array}{l}\text { Molecular Biology \& } \\
\text { Genetics }\end{array}$ & 4109 & 2825 & 1284 & $68.8 \%$ & $31.2 \%$ \\
\hline Clinical Medicine & 14592 & 9275 & 5317 & $63.6 \%$ & $36.4 \%$ \\
\hline Microbiology & 1612 & 1010 & 602 & $62.7 \%$ & $37.3 \%$ \\
\hline Neuroscience \& Behavior & 3473 & 2129 & 1344 & $61.3 \%$ & $38.7 \%$ \\
\hline Social Sciences, general & 2781 & 1609 & 1172 & $57.9 \%$ & $42.1 \%$ \\
\hline Multidisciplinary & 102 & 56 & 46 & $54.9 \%$ & $45.1 \%$ \\
\hline Plant \& Animal Science & 5733 & 2523 & 3210 & $44.0 \%$ & $56.0 \%$ \\
\hline Project average & 174425 & 143423 & 31002 & $82.2 \%$ & $17.8 \%$ \\
\hline
\end{tabular}

The total number of publications in journals of subscribed publishers increases faster than in other journals. The share of Russian publications in journals of unsubscribed publishers decreased from $18.7 \%$ in 2012 to $17.2 \%$ in 2016 . The total number of publications in subscribed journals increased from 2012 to 2016 by 29\%, while in journals of other publishers by $16 \%$. The distribution by ESI categories remains stable in analyzed period.

It should be considered that ESI categories do not include Arts \& Humanities, while the total number of Russian publication in AHCI increased twice from 2012 to 2016 (0.4\% of world output in 2012 and $0.9 \%$ in 2016), half of them annually published in subscribed journals $(0.3 \%$ of world output in journals of subscribed publishers in 2012 and $0.6 \%$ in 2016). 
The number of published articles correlates strongly with the number of downloaded articles, at least for publishers under consideration (Fig.6).

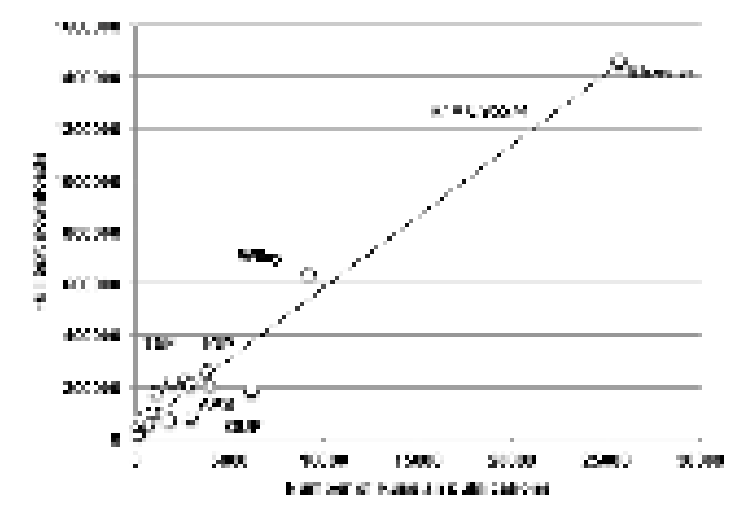

Fig. 6. Dependence of number of published papers (Russian Federation, 2012-2015, WoS CC) on number of downloaded articles. Correlation $\mathrm{r}=0.98$ (Pearson)

This coincides with the published data about dependence of research output on readership. However, it should be emphasized that all previous data has been reported either on a country, institutional or research area levels $[9,11,12]$. Here, for the first time, we report correlation between research output and readership for a set of separate packages of resources.

Dependence of publication output on total readership on an institutional level for the case of SPbSU is shown in Fig. 7.

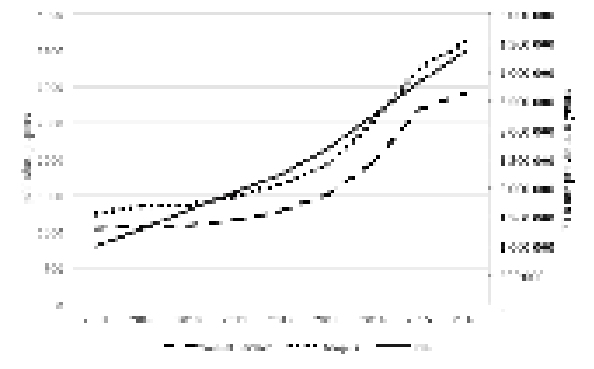

Fig. 7. Correlation of full-text downloads and published papers in Saint-Petersburg State University

$\mathrm{SPbSU}$ is having one of the best repertoires of subscribed resources in Russia, both thanks to state project and own university subscription. The list of subscribed resources increased from 
2008 to 2016 almost three times, including permanent access to purchased Elsevier backfiles. The number of full-text downloads increased during this period more than 4 times and correspondingly the publication output grew about three times both in WoS CC and Scopus. The increase in the number of downloads is determined not only by the more active use of resources but also by the growth in the number of users, and as a consequence, by an increase in the number of authors of scientific publications. According to SciVal data, the number of authors affiliated with SPbSU increased from 1669 in 2012 to 3704 in 2016 (the growth is more than 120\%). The analysis of SPbSU publication activity by Russian Index of Science Citation also demonstrates both growth of publications and number of authors together with the increasing of full-text downloads from RISC.

In our study we have experimentally obtained that the number of downloads in Russia depend directly on the number of articles available in the subscribed resources (Fig. 8).

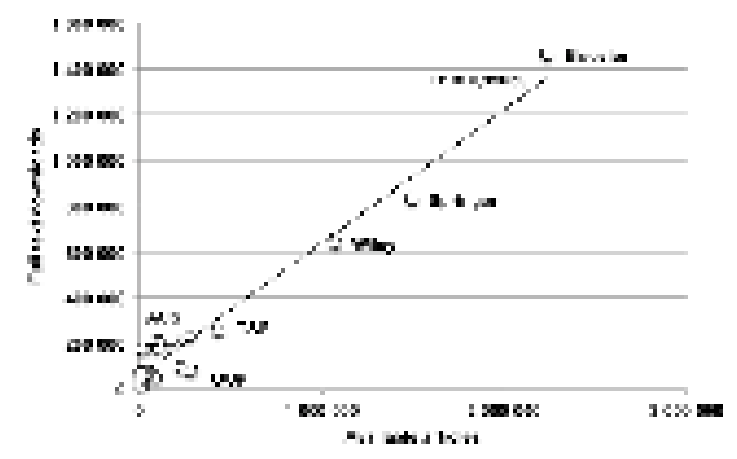

Fig.8. Dependence of full-text downloads on the number of available articles in resources under subscription. Correlation $\mathrm{r}=0.98$ (Pearson)

However, the reason of it is somewhat unclear. This fact could indicate that informetric laws (Pareto principle and Leimkuler curve) are not valid in this particular case when both packages of articles and ensemble of users were specially prepared. Noteworthy is that in our attempts to check the Lotka's law with usage statistics data obtained for the same packages, we also have found that the Lotka's law does not work in our case. Another explanation is that usage 
of electronic resources in Russian educational and research organizations is still far from saturation. For example, in Saint-Petersburg State University - the heaviest user in Russia, the number of full-text downloads per user amounted to about 100 articles in 2016, while in Great Britain such activity of users was characteristic for Consortium of University Research Libraries in 2010-2011 [36]. Thus, we are going to further investigate this in more detail.

To conclude, for the studied set of thoroughly selected resources and optimized numbers and particular lists of subscribers we can note a strong correlation ( $\mathrm{r}=0.98$, Pearson) between Russian research output and the number of articles available through the studied set resources subscribed within the Russian System of Information Support.

\section{Discussion}

Russian science reveals its specifics and differs essentially from that of the world total in terms of distribution of research output over research areas. More than a half of Russian publications in 2012-2016 are concentrated in two ESI research areas: Chemistry and Physics. This results from the specially organized $R \& D$ funding in Russia that covers five priority areas of Russian Science and Technology: Life Sciences; Industry of Nanosystems; ITC Systems; Environmental Management; Energy Efficiency, Energy Saving and Nuclear Energy and to a certain extent confirms the well-known fact that research output strongly correlates with funding. Since 2006 the state-funded subscription in Russia has been also focused at the five priority areas, so that the parameter of information support in those areas is high. In this study we have extended the field of our analysis to 22 ESI research areas. Results show that 16 full-text resources of current Russian national subscription, which were previously selected to fit five priority areas of Russian Science and Technology, reveal very high values of parameters of information support in all research areas of the ESI classificatory. If we take into account only 
journal packages of first seven world top multidisciplinary publishers: Elsevier, Springer-Nature, Wiley, T\&F, Sage, OUP and CUP we find that they cover more than $50 \%$ of world coverage in number of publications and citations. However, these high numbers are not sufficient to provide information in top research areas for Russian science, namely, Chemistry, Physics, Geoscience, Space Science and Computer Science. The lack of information in those areas is compensated due to resources published by world leading learning societies: ACS, RSC, APS, AIP, IoP, OSA, and IEEE. Those societies provide also the highest percentage of citations in key areas of Russian research: Physics and Chemistry. Therefore, one can conclude that despite a relatively low number of titles published by learning societies as compared to top multidisciplinary publishers, they are of key importance for the Russian and world research in Chemistry, Physics, Engineering and Computer Science. The numbers for 16 resources are as follows:

1. Coverage by the number of world publications varies over research areas from $85 \%$ in Chemistry to $48 \%$ in Microbiology and exceeds $55 \%$ for all ESI areas except Microbiology.

2. Coverage by the number of Russian publications - from $94 \%$ in Chemistry to $43 \%$ in Plant \& Animal Science and exceeds $60 \%$ for all ESI research areas except Social Science, general and Plant \& Animal Science.

3. Coverage by the number of world citations - from nearly $90 \%$ to $42 \%$.

4. Coverage by the number of citations in Physics: $30 \%$ are provided by APS articles and only $13 \%$ and $12 \%$, respectively, by Springer-Nature and Elsevier.

5. Open Access journals available via resources not included in national subscription compensate lack of information in such research areas as Microbiology and Plant \& Animal Science and make parameter of information support to exceed $65 \%$ for all ESI subject areas. 
For Russia, we report experimental data on strong correlation and linear dependence between research output and a number of full-text downloads from resources included in state-funded subscription. The linear dependence is confirmed both on national level and an example of a particular organization, namely, SPbSU - the second most productive university in Russia,

This fact is also supported by experimental temporal dependence of continues concentration of Russian articles in journals included in national subscription.

We also experimentally obtained linear dependence and strong Pearson correlation between the number of full-text downloads and the number of available articles in resources under subscription. This fact has not been reported in the literature yet and therefore needs further investigation.

Thus the existing state-funded subscription meets and satisfies the basic information needs of Russian science. The journals in the subscribed packages of world leading publishers investigated in this study are the main channels for publication of research results in Russia. Strong correlation of full-text downloads with research output in Russia demonstrates the necessity of further improvement of information support in all research areas, especially those which are insufficiently presented in the world information space (social sciences and humanities).

Results obtained demonstrate applicability of InCites instruments for collection development and evaluation. The study was performed in Russian research and information surroundings, however, results and methods could be easily adjusted to any set of resources presented in WoS CC.

\section{Acknowledgements}

Input of one of the authors is supported by Ministry of Education and Science of the Russian Federation, RFMEFI60316X0019. 


\section{REFERENCES}

1. Excellence in Research for Australia (ERA). Available at: http://www.arc.gov.au/excellence-research-australia (Retrieved 01.07.2017).

2. Research Excellence Framework (REF). Available at: http://www.ref.ac.uk/ (Retrieved 01.07.2017)

3. AERES, the Agence d'évaluation de la recherche et de l'enseignement supérieur. Available at: http://www.aeres-evaluation.fr/ (Retrieved 01.07.2017).

4. VQR (Valutazione della Qualità della Ricerc $a$, Evaluation of Research Quality). Available at: https://researchassessment.fbk.eu/vqr_evolution (Retrieved 01.07.2017).

5. Information and analytical data on the results of monitoring of efficiency of higher educational institutions. Available at: http://indicators.miccedu.ru/monitoring/?m=vpo (In Russ.). (Retrieved 01.07.2017).

6. Sandstrom, U. "Research quality and diversity of funding: A model for relating research money to output of research". Scientometrics. 79, 2 (2009): 341-349. URL: http://link.springer.com/article/10.1007/s11192-009-0422-2/. (Retrieved 01.07.2017)). DOI:10.1007/s11192-009-0422-2

7. Xianwen Wang, Di Liu, Kun Ding, Xinran Wang. "Science funding and research output: a study on 10 countries". Scientometrics. 91, 2 (2012): 591-599. Available at: http://link.springer.com/article/10.1007/s11192-011-0576-6/ (Retrieved 01.07.2017). DOI:10.1007/s11192-011-0576-6

8. Leydesdorff L., Wagner C.. "Macro-level indicators of the relations between research funding and research output”. Journal of Informetrics. 3, 4 (2009): 353-362. Available at: http://www.sciencedirect.com/science/article/pii/S1751157709000418/ https://doi.org/10.1016/j.joi.2009.05.005. (Retrieved 01.07.2017). 
9. Razumova I. K. “Consortial and national subscriptions in Russia and worldwide. Support with and use of scholarly information and research output. Latest trends”. In: Materialy VI Vserossiyskoy nauchno-prakticheskaya konferentsii "Fondy bibliotek v tsifrovuyu epokhu: traditsionnye i elektronnye resursy, komplektovanie, ispolzovanie". SanktPeterburg, Rossiya, $2016=$ Proceedings of the sixth All-Russia scientific and practical conference "Library funds in digital age: traditional and electronic resources, acquisition and use”. St. Petersburg. Russia. 2016. Available at: http://www.nlr.ru/tus/20160328/present/razumova_3003.pdf. (In Russ.) (Retrieved 01.07.2017).

10. Moed, H. F. "Statistical relationships between downloads and citations at the level of individual documents within a single journal". Journal of the American Society for Information Science and Technology. 56, 10 (2005): 1088-1097. DOI:10.1002/asi.20200

11. Jung Y., Kim J., So M., Kim H. "Statistical relationships between journal use and research output at academic institutions in South Korea". Scientometrics. 103, 3 (2015): 751-777. Available at: http://link.springer.com/article/10.1007/s11192-015-1563-0/ (Retrieved 01.07.2017). DOI:10.1007/s11192-015-1563-0.

12. Kuznetsov A.Yu., Razumova I.K. "Scientific publications as criteria of science development”. Rossiyskie nanotekhnologii. Vol. 2, No. 11-12 (2007): 28-39. (In Russ.) (Retrieved 12.05.2017).

13. Library Budgets Predictions for 2016 - Publishing Communication Group, Available at: http://www.pcgplus.com/wp-content/uploads/2016/05/Library-Budget-Prediction-2016Final.pdf . (Retrieved 01.07.2017).

14. Razumova I.K., Kuznetsov A.Yu., Kirillova O.V. "Information support of Russian universities in key research areas". Integration of Education. 27, 3 (2017): 505-521. 505- 
521. DOI: 10.15507/1991-9468.088.021.201703.505-52. (published online on 29.09.2017)

15. Evans, P. "The Impact of Science in Asia (especially China) on the Further Globalisation of Related Information Flows. A publisher's View”. Presentation at ICOLC Fall 2006 (8th European Meeting), October 2006. Available at http://www.aepic.it/gli-ateneiitaliani-per-lopen-access-verso-laccesso-aperto-alla-letteratura-di-ricerca/?id=170\&cf $=7$.

16. Kuznetsov A.Yu., Razumova I.K. "System of information support of science and higher education in Russia”. Rossiyskie nanotekhnologii. Vol.2, No. 11-12 (2007): 19-27. (In Russ.) (Retrieved 12.05.2017).

17. Kuznetsov A. Yu., Razumova I. K. "Selling to the BRIC: Russia". Learned Publishing, 24, 2 (2011): 139-144(6).

18. Kouznetsov, A., Litvinova, N. \& Razumova, I. "National Electronic Information Consortium as the Leader of Cooperative Acquisition of E-resources in Russia”, San Juan, Puerto Rico, IFLA 2011. Available at: https://www.ifla.org/past-wlic/2011/113kouznetsov-en.pdf/ (Retrieved 01.07.2017).

19. Petrov A.N. "Information support of science and education". In: The $3 r d$ NEICON International Conference «Electronic Resources for Research and Education: Development, Promotion and Use», Halkidiki, Greece 2016. Available at: conf.neicon.ru/materials/16-Overseas2015/150929-03-Petrov.ppt. (In Russ.) (Retrieved 01.07.2017)

20. Evstigneeva G.A. "National access to international databases within the Federal Target Program "Research and Development in Priority Fields of the Science and Technology Complex of Russia for the Years 2014-2020"'. Nauchnye i tekhnicheskie biblioteki. 5 (2016): 29-43. (In Russ.) (Retrieved 01.07.2017). 
21. Bullington, J. "About ICOLC and the ICOLC Statement on the Global Economic Crisis and Its Impact on Consortial Licenses”, Collaborative Librarianship. 1 (2009): 156-161. Available at: http://digitalcommons.du.edu/collaborativelibrarianship/vol1/iss4/7. (Retrieved 01.07.2017).

22. Zervas M, Heraki M. "The Challenge of Creating the Cyprus Academic Library Consortium (CALC): Impacts and Benefits. 2017’. Expanding Perspectives on Open Science: Communities, Cultures and Diversity in Concepts and Practices. L. Chan and F. Loizides (Eds.). Available at: http://lekythos.library.ucy.ac.cy/bitstream/handle/10797/22303/zervas2.pdf?sequence=1. (Retrieved 01.07.2017). DOI:10.3233/978-1-61499-769-6-283.

23. Razumova I.K. "Journals and books. Subscription to electronic resources in Russia and worldwide: the 2016-2017 survey results”. In: Materialy VII Vserossiyskoy nauchnoprakticheskaya konferentsii "Fondy bibliotek v tsifrovuyu epokhu: traditsionnye $i$ elektronnye resursy, komplektovanie, ispolzovanie". Sankt-Peterburg, Rossiya, $2017=$ Proceedings of the $7_{\text {th }}$ All-Russian scientific and practical conference "Library funds in digital age: traditional and electronic resources, acquisition and use”. St. Petersburg. Russia. 2017. Available at: http://www.nlr.ru/tus/20170327/prezent/23.pdf. (In Russ.) (Retrieved 01.07.2017).

24. Ewa Dzurak, Kerry A. Falloon, Jonathan Cope. "Evaluating and planning for interdisciplinary collection development: a case of an East Asian collection at the College of Staten Island Library", Collection Building, Vol. 34 Issue: 2 (2015): 51-58, https://doi.org/10.1108/CB-01-2015-0002

25. Cheryl D. Bain, April L. Colosimo, Tara Mawhinney \& Louis Houle. "Using WorldShare Collection Evaluation to Analyze Physical Science and Engineering Monograph Holdings 
by Discipline". Collection Management, $41: 3$ (2016): 133-151, DOI:

$10.1080 / 01462679.2016 .1208132$

26. Gureyev V, Mazov N. "Detection of Information Requirements of Researchers Using Bibliometric Analyses to Identify Target Journals". Information technology and Libraries, December 2013: 66-77

27. Gureev V.N., Mazov N.A. "Assessment of the relevance of journals in research libraries using bibliometrics (a review)”. Scientific and Technical Information Processing. 42. № 1 (2015): 30-40.

28. Gureev V.N. "Bibliometric analysys as the basis for formation of repertoire of scholarly periodicals in libraries". Thesis for the degree of Candidate of Pedagogical Sciences / Moscow State Institute of Culture. Moscow, 2015

29. Evans, G.E., "Management issues of cooperative ventures and consortia in the USA. Part one". Library Management. 23, 4/5 (2002): 213-226. Available at: http://dx.doi.org/10.1108/01435120210429943 (Retrieved 01.07.2017). DOI: $10.1108 / 01435120210429943$

30. Kinner, L., Crosetto, A., "Balancing Act for the Future: How the Academic Library Engages in Collection Development at the Local and Consortial Levels". Journal of Library Administration. 49, 4 (2009): 419-437. Available at: http://www.tandfonline.com/doi/abs/10.1080/01930820902832561. (Retrieved 01.07.2017). DOI: 10.1080/01930820902832561

31. Machovec G. "Library Networking and Consortia". Journal of Library Administration. 57, 5 (2017): 577-584. URL: http://www.tandfonline.com/doi/abs/10.1080/01930826.2017.1326266?journalCode=wjla 20. (Retrieved 01.07.2017). DOI:10.1080/01930826.2017.1326266 
32. Pan, D., Fong, Y. "Return on Investment for Collaborative Collection Development: A Cost-Benefit Evaluation of Consortia Purchasing". Collaborative Librarianship. 2, 4 (2010): 183-192. Available at:

http://digitalcommons.du.edu/cgi/viewcontent.cgi?article=1232\&context=collaborativelib rarianship. (Retrieved 01.07.2017).

33. Kelly, B., Hamasu, C., Jones, B., “Applying Return on Investment (ROI) in Libraries”. Journal of Library Administration. 52, 8 (2012): 656-671. Available at: http://www.tandfonline.com/doi/abs/10.1080/01930826.2012.747383. (accessed 01.07.2017). DOI: 10.1080/01930826.2012.747383

34. Hormia-Poutanen K., Xenidou-Dervou C., Kupryte R., Stange K., Kuznetsov A., Woodward H. "Consortia in Europe: Describing the Various Solutions through Four Country Examples “ Library Trends. 54, 3 (2006): 359-381.

35. Piwowar, P., Larivière, A.M., Norlander, F., West, H. “The State of OA: A large-scale analysis of the prevalence and impact of Open Access articles”. PeerJ Preprints, https://doi.org/10.7287/peerj.preprints.3119v1 CC BY 4.0 Open Access | rec: 2 Aug 2017, publ: 2 Aug 2017.

36. The Society of College. National and University Libraries. SCONUL. Annual Library Statistics. 2010-2011. SCONUL. (2012). Available at: http://www.sconul.ac.uk/sites/default/files/documents/ALS1011.pdf/ (Retrieved: 01.07.2017). 
